\title{
Complexidade, conformidade e arrecadação tributária *
}

\author{
Otávio Gomes Cabello ** \\ Silvio Hiroshi Nakao ****
}

\begin{abstract}
Resumo
Este trabalho objetiva apresentar qual é o custo de conformidade tributário nos Estados brasileiros e examinar se a complexidade tributária afeta os custos de conformidade e se estes afetam a arrecadação. Os resultados: (a) evidenciam que empresas de todos os Estados brasileiros possuem um custo de conformidade do ICMS, considerando apenas mão de obra, na média de 1,5\% da obrigação principal; e (b) sugerem que uma maior complexidade tributária leva a maiores custos de conformidade, que por sua vez aumentam a arrecadação tributária. Isso significa que o aumento da complexidade tributária pode tornar o sistema econômico menos eficiente e competitivo, pois o aumento na complexidade da legislação precisa ser acompanhado de sanções que forcem o contribuinte a incorrer em custos adicionais, de modo a fazer com que haja um aumento da arrecadação. Este trabalho contribui com o debate sobre a simplificação tributária e seus efeitos econômicos; e com a literatura sobre custos de conformidade.
\end{abstract}

Palavras-chave: Complexidade tributária, Conformidade tributária, Arrecadação tributária, Custo de conformidade.

\section{Abstract \\ Complexity, compliance and tax collection}

This paper aims to present the cost of tax compliance in Brazilian states and to examine whether tax complexity affects compliance costs and whether they affect collection. The results show that: (a) companies from all Brazilian states incur an ICMS compliance cost, considering tax employees alone, at an average of $1.5 \%$ of the ICMS; and (b) that greater tax complexity leads to higher compliance costs, which lead to an increase in tax collection. This means that increasing tax complexity can make the economic system less efficient and competitive, as the increase in the complexity of legislation must be accompanied by taxpayer enforcement to incur additional costs in order to increase collection. This paper contributes to the debate about tax simplification and its economic effects as well as to the literature on compliance costs.

Keywords: Tax complexity, Tax compliance, Tax collection, Compliance cost.

JEL: H21, H25, H29.

\section{Introdução}

A literatura aponta que uma maior complexidade tributária pode gerar uma menor conformidade com a legislação (Cox; Eger, 2006) e consequente evasão (Richardson, 2006), em função da maior dificuldade que os contribuintes têm para compreendê-la e cumpri-la (Saad, 2014).

Porém, dado que são esperadas sanções para a não conformidade (Allingham; Sandmo, 1972), é possível esperar que os contribuintes incorram em custos superiores para atender à maior complexidade tributária. De fato, Gupta e Mills (2003) mostram que a complexidade pode afetar os

\footnotetext{
${ }^{*}$ Artigo recebido em 25 de setembro de 2019 e aprovado em 18 de fevereiro de 2021.

** Professor Doutor da Faculdade de Ciências Aplicadas da Universidade Estadual de Campinas (FCA-Unicamp), Limeira, SP, Brasil. E-mail: ocabello@unicamp.br. ORCID: https://orcid.org/0000-0003-4933-512X.

${ }^{* * *}$ Professor Associado da Faculdade de Economia, Administração e Contabilidade da Universidade de São Paulo (FEA-RP/USP), Ribeirão Preto, SP, Brasil. E-mail: shnakao@usp.br. ORCID: https://orcid.org/0000-0003-3976-8407.
} 
custos de conformidade das empresas, observando a complexidade dada pelo fato de as legislações e o imposto de renda nos Estados Unidos serem estaduais.

A maior complexidade tributária tem por objetivo uma maior eficiência na arrecadação, seja porque a descentralização da legislação permitiria maior autonomia e, em consequência, melhor adaptação às realidades locais, seja porque é necessária para acompanhar as mudanças nos negócios ao longo do tempo. Com a passagem do tempo, contribuintes podem encontrar meios de planejamento tributário, com a própria evolução dos negócios, que podem forçar o fisco a aperfeiçoar a legislação.

Esse raciocínio nos leva a pensar que a complexidade tributária não afeta diretamente a arrecadação, porque é necessário que o contribuinte incorra em custos para atender à maior complexidade e evitar sanções, o que por sua vez afetaria a arrecadação.

Entretanto, a literatura não é suficientemente clara sobre esse processo que liga a complexidade tributária à arrecadação. Conhecer o mecanismo que estabelece essa ligação é relevante porque está diretamente ligada à política tributária de qualquer país. Apesar de relevante, pesquisas nesse assunto são escassas em função de baixa disponibilidade de dados para verificação empírica.

Porém, conseguimos ter acesso a dados sobre a evolução de dispositivos legais no Brasil, o que abriu a oportunidade de realizarmos testes empíricos para verificar essa relação entre complexidade tributária, custo de conformidade e arrecadação. Este trabalho objetiva apresentar qual é o custo de conformidade tributário nos Estados brasileiros e examinar se a complexidade tributária afeta os custos de conformidade e se, por sua vez, estes afetam a arrecadação, aproveitando particularidades da tributação no Brasil.

Um dos tributos arrecadados no Brasil é o ICMS, que possui 27 legislações tributárias diferentes por se tratar de um imposto cobrado por Estados e Distrito Federal (DF). Essa característica já é representativa de complexidade, tal como Gupta e Mills (2003) examinaram os Estados Unidos com legislações de imposto de renda estaduais. Há de se considerar também o dinamismo com que essas normas são alteradas. Segundo Oliveira (2012), em outubro de 2012 foram feitas 20 alterações por dia na legislação do ICMS. Alterações em grandes quantidades que podem ser observadas diariamente nas publicações dos Diários Oficiais da União e Estados. Essa particularidade do Brasil permite observar com maior nitidez a complexidade tributária e suas consequências.

Nós utilizamos dados de quantidade de dispositivos legais do ICMS por Estado como proxy para a complexidade tributária e testamos com dados sobre remunerações de profissionais da área contábil e fiscal obtidas da Relação Anual de Informações Sociais (RAIS), como proxy para custos de conformidade. Assim, evidenciamos os custos de conformidade por Estado. Em seguida, verificamos a relação entre custos de conformidade e arrecadação, por meio dos valores arrecadados de ICMS extraídos do Conselho Nacional de Política Fazendária (CONFAZ).

Os resultados sugerem que uma maior complexidade tributária leva a maiores custos de conformidade, que por sua vez aumentam a arrecadação tributária. Esses resultados têm implicações econômicas. Um aumento na complexidade da legislação precisa ser acompanhado de sanções que forcem o contribuinte a incorrer em custos adicionais, de modo a fazer com que haja um aumento da arrecadação. De outro modo, o aumento da complexidade pode não resultar em aumento da 
arrecadação. Isso significa que o aumento da complexidade tributária pode tornar o sistema econômico menos eficiente e competitivo, o que pode colaborar para o debate sobre a simplificação tributária.

Assim, este trabalho contribui com o debate sobre a simplificação tributária e seus efeitos econômicos. Também contribui com a literatura sobre custos de conformidade, ao estabelecer relação com a complexidade tributária e a arrecadação, lacuna ainda não preenchida na literatura na área tributária.

Do ponto de vista prático, este estudo pode contribuir como justificativa para o estabelecimento de políticas econômicas que resultem em uma simplificação nos modelos de tributação, possam melhorar a eficiência da economia.

\section{Revisão da literatura e formulação das hipóteses}

\subsection{Complexidade tributária}

A complexidade tributária, de acordo com Saad (2014), pode assumir diversas formas, pois pode ser influenciada por complexidades computacionais, por forma, por conformidade, por regras, por procedimentos ou por legibilidade.

De acordo com Oliver e Bartley (2005) os custos de arrecadação de tributos incluem custos de administração do governo e os custos de conformidade dos contribuintes no cumprimento de suas obrigações nos termos da lei. Os autores afirmam que se um sistema tributário estiver associado a níveis excessivos de custos de conformidade, a economia não desempenhará sua curva de possibilidades de produção; e complementam afirmando que a complexidade cumulativa, ou seja, seguidas mudanças na legislação tributária, mesmo mudanças relativamente simples, podem impedir coletivamente a capacidade dos contribuintes de entender os requisitos da lei sem precisar procurar assistência profissional. Sendo assim, observa-se que mesmo que mudanças na legislação sejam simples, o efeito para os contribuintes é de majoração no custo de conformidade.

Nesse sentido, formulou-se a seguinte hipótese:

H1: Quanto maior a complexidade tributária, maior o custo de conformidade.

\subsection{Pesquisas em custo de conformidade}

O custo dos tributos pode ser estruturado em três custos, conforme Bertolucci (2001): (a) custo dos impostos, taxas e contribuições, ou seja, a obrigação principal; (b) custo de distorção, que é representado pelas mudanças na economia que afetam diretamente o produto ou tributos do mesmo; e (c) custos operacionais tributários, que estão relacionados aos recursos aplicados para o cumprimento das regras tributárias, previstas no sistema tributário ou nas regras de cada tributo. Este último pode ser divido em Custos de Administração e Custo de Conformidade Tributária. A Figura 1 evidencia a composição do custo de tributos. 
Figura 1

Custo dos tributos

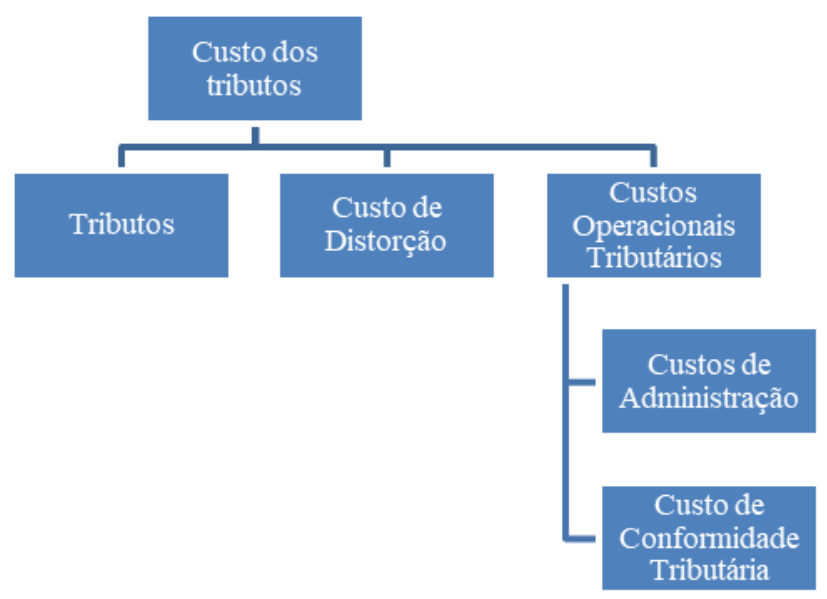

Fonte: Bertolucci (2001) adaptado.

Paes (2007) aponta que o custo para cumprimento da obrigação tributária, também chamado de custos administrativos indiretos, possui formas variadas, por incluir o custo do tempo gasto no preenchimento de formulários e declarações, custo de serviços contábeis e jurídicos, além da guarda e manutenção de livros fiscais e documentos.

No Brasil, atualmente, a guarda das informações encontra-se em forma digital. No entanto, existe um custo para sua elaboração e manutenção. Isso significa dizer que há gastos com empresas especializadas em atualização da informação tributária.

Bertolucci (2001) realizou pesquisa com 200 empresas abertas no Brasil, mas apenas 25 questionários foram considerados satisfatórios, e constatou que aproximadamente de $2,5 \%$ a $3 \%$ da receita líquida dessas empresas é para remunerar o custo do cumprimento da legislação tributária. A natureza do seu procedimento metodológico adotado não objetiva a generalização, mas sinaliza que os custos de conformidade podem ser materiais nas empresas.

Cavalcanti Filho (2009) realizou um estudo de caso em um empreendimento de construção civil, localizado no município de Manaus, Estado do Amazonas. Os resultados apontaram para um custo de conformidade de 7,26\% em relação ao resultado. Esse resultado foi obtido a partir de percentuais discricionários aplicados às despesas do empreendimento que tivessem relação com o cumprimento de obrigações tributárias (principal e acessória). Segundo a pesquisa, os percentuais foram estabelecidos pelo próprio departamento ao qual a despesa estava relacionada.

Ferreira (2012) propôs um modelo de mensuração dos custos de conformidade tributária. O estudo foi baseado na metodologia design research, apresentado como ferramenta que gera informações por meio do custeio baseado em atividades. Foi utilizada uma empresa gaúcha do terceiro setor como caso para teste do modelo e identificou-se que, de acordo com o modelo sugerido, a empresa em questão gasta $\mathrm{R} \$ 3,25$ para cada $\mathrm{R} \$ 1,00$ pago de tributos, sinalizando, assim, que os gastos com o custo de conformidade podem ser maiores que a própria obrigação tributária principal. 
A pesquisa de Miyoshi e Nakao (2012) evidenciou os riscos relacionados à conformidade tributária. Utilizou como estudo de caso uma empresa do Estado de São Paulo; e realizou a implantação de um modelo de gerenciamento de riscos tributários relacionados ao ICMS. Os resultados, baseados no método de gerenciamento de riscos sugerido pelo COSO (Committee of Sponsoring Organizations) que trabalha com probabilidades de ocorrência, apontam que houve uma redução média geral de 33,92\% nos riscos tributários após a implantação do gerenciamento de riscos. Outro achado da pesquisa foi que a aversão ao risco se refere principalmente à probabilidade de ser fiscalizada, e não exatamente ao impacto dos riscos tributários a que a empresa está exposta, o que pode indicar que as empresas, muitas vezes, incorrem em custos de conformidade para se evitar uma fiscalização.

Souza, Balbino e Miranda (2013) realizaram um estudo de caso de uma empresa comercial de Recife para indicar o custo de conformidade no processo de planejamento tributário. Os resultados apontam que, considerando os custos de conformidade no processo de planejamento tributário de constituição de filiais em vários Estados, pode-se obter um incremento tributário de R \$22 mil. Notase que este estudo evidencia o custo de conformidade especificamente para a prática do planejamento tributário proposto e não das operações cotidianas das companhias.

Lima (2013) efetuou um estudo sobre redução nos custos de conformidade tributária a partir da implantação do Sistema Público de Escrituração Digital (SPED). A pesquisa foi realizada em 20 empresas brasileiras de tamanhos e segmentos distintos, buscando capturar três vertentes de custo de conformidade: pessoas, tecnologia e contratação de serviços de consultoria específica. Os resultados indicaram que o SPED provocou um aumento dos custos de conformidade temporários e permanentes, em função da estratégia de implementação definida e aplicada unicamente pela administração pública. Os resultados também apontaram que os investimentos privados direcionados para a conformidade ao SPED não formam os mesmos aplicados pela administração pública para a vigência do sistema, o que evidencia uma transferência dos custos de administração para os custos de conformidade dos contribuintes. É fato que os resultados, pelos procedimentos metodológicos adotados, não são conclusivos para extensão dos resultados nas empresas em geral. No entanto, é um indicativo de que, mesmo com a vigência do SPED, que visava a redução das obrigações assessórias, não houve uma redução dos custos de conformidade tributária.

Constata-se que a maioria das pesquisas apresentadas é feita por meio de estudo de caso, o que pode ser justificado pela maior capacidade de coleta de dados para mensuração dos custos de conformidade.

Pesquisas com diversas empresas podem ser encontradas com mais frequência fora do Brasil. Slemrod e Blumenthal (1996), após efetuarem pesquisa com 1.300 grandes empresas norteamericanas em 1992, afirmaram que os maiores custos tributários estaduais são em função da não conformidade dos sistemas tributários estaduais.

Outras pesquisas internacionais sobre custo de conformidade, como as de Kaplow (1996), Slemrod e Venkatesh (2002), Evans e Tran-nam (2002), Kopezuk (2006) e de Das-Gupta (2006) apontam para o peso do custo da conformidade em companhias de diversos países. O que evidência que essa é uma realidade mundial e não apenas brasileira, guardadas as devidas proporções em relação à economia, estabilidade institucional e cultura de cada país. Claro está que esta pesquisa não pretende tecer comparações dos sistemas tributários e suas complexidades. 
O relatório da PWC (Price Waterhouse and Cooper) (2017) sobre pagamento de impostos, apontou que os contribuintes brasileiros, no ano de 2014, precisaram de 2.038 horas para cumprir obrigações assessórias. Segundo o estudo, o Brasil, pela primeira vez desde que o relatório anual é elaborado (2007), conseguiu reduzir a quantidade de horas (562 horas) em função da implantação de sistemas digitais para envio de informações ao fisco.

O estudo da PWC (2017) revela que o contribuinte brasileiro, das 2.038 horas dispendidas com o cumprimento de obrigações assessórias, 486 são para tributos sobre o lucro, 363 tributos trabalhistas e 1.189 tributos sobre o consumo. Sendo assim, observa-se que o ICMS, Programa de Integração Social (PIS), Contribuição para o Financiamento da Seguridade Social (Cofins), Imposto sobre Produto Industrializados (IPI) e Imposto sobre Serviço de Qualquer Natureza ISSQN são os tributos que consomem mais tempo para conformidade.

Observa-se, pela revisão da literatura, que as empresas incorrem em custos significativos de conformidade para atender às demandas da legislação ou para evitar riscos decorrentes da não conformidade. Se essas demandas da legislação são mecanismos que evitam a redução da arrecadação em função de novos mecanismos de planejamento tributário por parte dos contribuintes ou buscam aumentar a eficiência da arrecadação, podemos esperar que esses custos de conformidade dos contribuintes tenham efeito sobre a arrecadação de tributos. Assim, estabelecemos a seguinte hipótese:

H2: Quanto maior o custo de conformidade, maior a arrecadação.

\section{Método}

Como meio de observar as relações propostas nas duas hipóteses do trabalho, procuramos aproveitar as características da tributação brasileira e a disponibilidade de dados para executar os testes empíricos. Sendo assim, pela disponibilidade de dados, foi selecionado o ICMS para compor a base de dados, e não selecionamos os demais tributos diretos e indiretos incidentes sobre as empresas.

A tributação brasileira é caracterizada por forte tributação sobre o consumo, especialmente sobre o valor agregado, com legislação descentralizada, realizada pelos Estados da Federação. O ICMS, além de ser o tributo mais representativo na carga tributária do país (RFB, 2017), é o que mais apresenta complexidade em função da quantidade de dispositivos legais. Basta observar a quantidade de regulamentos que vigoram nos 27 Estados Brasileiros e no DF, sem contar outros atos que são publicados diariamente nos Diários Oficiais.

Nesse sentido, coletamos os seguintes dados dos anos de 2011 a 2016:

- Quantidade de dispositivos legais do ICMS por Estado. Dados obtidos por meio da empresa Systax Sistemas Fiscais Ltda., que fez um mapeamento dos dispositivos legais do ICMS nos Estados Brasileiros e no DF. Nessa variável, foi considerado cada dispositivo legal constante nas leis de ICMS dos Estados Brasileiros, não obrigações acessórias em específico;

- Número de contadores, auxiliares de contabilidade, e respectivas remunerações médias mensais. Dados obtidos dos microdados da Relação Anual de Informações Sociais (RAIS), sob o Código Brasileiro de Ocupação (CBO) 2522-10 (Contador), 2522-05 (Auditor - contadores e afins), 4131-10 (auxiliar de contabilidade) e 4131-15 (auxiliar de faturamento). O CBO 4110-05 (auxiliar de folha de pagamento) não foi comtemplado, pois não atua com conformidade do ICMS. Foram filtrados os dados apenas de Entidades Empresariais, código de natureza jurídica 2, ou seja, 
organizações públicas (direta, indireta) não forma comtempladas. Da remuneração média mensal, foi calculado e somado o $13^{\circ}, 1 / 3$ de férias, bem como $40 \%$ de encargos sociais. Na coleta dessa variável a pesquisa considerou que tanto colaboradores internos de empresas como colaboradores de empresas de serviços contábeis compõem a amostra, pois ambos trabalham para a conformidade do ICMS;

- Número de empresas por Estado, excluindo as optantes do Simples Nacional, pois essas devem seguir a Lei Complementar 123/06 e alterações posteriores. Dados extraídos do caderno de Estatística do Cadastro Central de Empresas publicado pelo IBGE (2017), referente aos anos de 2011 a 2016. Foram selecionadas as seguintes atividades, pois são contribuintes do ICMS: (a) Agricultura, pecuária, produção florestal, pesca e aquicultura; (b) Indústrias extrativas; (c) Indústrias de transformação; (d) Eletricidade e gás; (e) Comércio; reparação de veículos automotores e motocicletas; (f) Alojamento e alimentação; e (g) Transporte, armazenagem e correio. Cabe destacar que algumas atividades mencionadas não são tributadas pelo ICMS, mas não havia dados individualizados disponíveis. A quantidade de empresas do Simples Nacional foi extraída da Receita Federal do Brasil (2018) e foram consideradas apenas empresas dos Anexos I e II, pois são as empresas contribuintes do ICMS, correspondentes às empresas do Cadastro Central de Empresas;

- Arrecadação do ICMS por estado, mais o DF. Dados extraídos do Conselho Nacional de Política Fazendária (CONFAZ) (2018); (2017);

- Carga tributária do ICMS no Brasil. Dados coletados da Receita Federal do Brasil

- Horas despendidas para conformidade da legislação tributária. Dados extraídos do relatório da PWC (2017).

- Produto Interno Bruno. Dados extraídos do IBGE (2017b). O PIB dos Estados, para o ano de 2016, foi replicado do ano de 2015, pois até o momento desta pesquisa o IBGE não divulgou os dados de 2016;

- Alíquota do ICMS. Dados extraídos do CONFAZ (2018b).

Após a coleta de dados, foi calculado o Custo de Conformidade Total por estado do período de 2011 a 2016 da seguinte forma:

$$
\begin{aligned}
& \text { Custo de Conformidade }=\left(\left(\text { Salário Médio Mensal }+13^{\circ} \text { salário }+\frac{1}{3} \text { de férias }+\right.\right. \\
& 40 \% \text { de encargos }) \times \text { Número de Profissionais }) \times \\
& \text { Proporção do número de horas despendidas com a conformidade do ICMS }
\end{aligned}
$$

Em que:

Proporção do número de horas despendidas com a conformidade do ICMS, foi considerado que das 1.189 horas despendidas com Consumption Taxes (PWC, 2017), 642,89 horas (54,07\%) seriam com ICMS, uma vez que existem 5 tributos sobre o consumo. Essa proporção foi calculada considerando que o ICMS representou $20,37 \%$ da carga tributária total (RFB, 2017) dos 37,67\% dos indiretos, ou seja, 54,07\%.

Calculado o Custo de Conformidade do ICMS para cada estado, foi calculado o Custo de Conformidade do ICMS por disposto legal, ponderado pela quantidade de empresas (exceto as optantes do Simples) para uma menor dispersão. Para tanto foi realizada a seguinte forma: 
Custo de Conformidade por Dispositivo Legal = (Custo de Conformidade $\div$ Quantidade de Empresas) $\div$ Quantidade de Dispositivos Legais

Com posse dos dados de arrecadação e quantidade de dispositivos legais, foi calculada a Arrecadação por Dispositivo Legal, também ponderada pela quantidade de empresas (exceto as optantes pelo Simples).

Arrecadação por Dispositivo Legal = (Arrecadação do ICMS $\div$ Quantidade de empresas $) \div$ Quantidade de Dispositivos Legais

Por fim, foi calculada a representação do Custo de Conformidade por Dispositivo Legal com a Arrecadação por Dispositivo Legal:

\section{Representação do Custo de Conformidade por Dispositivo Legal com a Arrecadação por Dispositivo Legal $=$ Custo de Conformidade por Dispositivo Legal $\div$ Arrecadação por Dispositivo Legal}

Após todos os cálculos, foi realizado um ranqueamento dos Estados com o objetivo de observar quais Estados possuem maior custo de conformidade, maior arrecadação por dispositivo legal e conhecer qual a representatividade do custo de conformidade na arrecadação por dispositivo legal.

Realizado o ranqueamento, a pesquisa utilizou-se de três modelos de regressão linear com dados em painel, efeitos fixos, cujas variáveis estão apresentadas no Quadro 1, para testar a relação entre o custo de conformidade e dispositivos legais com a arrecadação do ICMS dos Estados, e se a variação dos dispositivos legais impacta na variação no custo de conformidade de modo a dar maior robustez aos resultados. Foi utilizado o modelo de regressão com efeito fixo no tempo, por se tratar de dados em painel, e segue os modelos da literatura internacional. O modelo foi estimado pelo método de Mínimo Quadrado Generalizados (GLS).

Quadro 1

Variáveis explicativas

\begin{tabular}{|c|c|c|c|}
\hline Variáveis & Fórmula & Tipo de variável & Fonte \\
\hline $\begin{array}{c}\text { Arrecadação do ICMS } \\
\text { (AT) }\end{array}$ & $\begin{array}{l}\text { Arrecadação total do ICMS dos Estados de } \\
2011 \text { a } 2016\end{array}$ & $\begin{array}{c}\text { Dependente nos } \\
\text { Modelos I e III }\end{array}$ & CONFAZ (2018) \\
\hline $\begin{array}{l}\text { Custo de Conformidade } \\
\text { (CustoConf) }\end{array}$ & $\begin{array}{l}\text { (Salário Médio Mensal de dezembro de } \\
\text { cada ano }+13^{\circ} \text { salário }+1 / 3 \text { de férias }+ \\
40 \% \text { de encargos }) \times \text { Número de } \\
\text { Profissionais }) \times \text { Proporção do número de } \\
\text { horas despendidas com a conformidade do } \\
\text { ICMS }]\end{array}$ & $\begin{array}{l}\text { Independente nos } \\
\text { Modelos I e III } \\
\text { Dependente no } \\
\text { Modelo II }\end{array}$ & $\begin{array}{l}\text { Criada pelos } \\
\text { autores }\end{array}$ \\
\hline $\begin{array}{l}\text { Variação do Número de } \\
\text { Dispositivos Legais } \\
\text { (VARDL) }\end{array}$ & $\begin{array}{l}\left(\mathrm{N}^{\mathrm{o}} \text { de Dispositivos Legais } \mathrm{x} / \mathrm{N}^{\mathrm{o}} \text { de }\right. \\
\text { Dispositivos Legais } \mathrm{x}-1)-1\end{array}$ & Independente & $\begin{array}{l}\text { Criada pelos } \\
\text { autores }\end{array}$ \\
\hline PIB & $\begin{array}{l}\text { Participação percentual do PIB do estado } \\
\text { no PIB geral. }\end{array}$ & Controle & IBGE (2017b) \\
\hline $\begin{array}{l}\text { Número de Empresas } \\
(\text { EMP })\end{array}$ & $\begin{array}{l}\text { Quantidade de empresas, exceto as } \\
\text { optantes pelo Simples Nacional }\end{array}$ & Controle & $\begin{array}{l}\text { IBGE (2017) } \\
\text { adaptada pelos } \\
\text { autores } \\
\end{array}$ \\
\hline $\begin{array}{l}\text { Alíquota de ICMS } \\
\text { (Alíquota) }\end{array}$ & $\begin{array}{l}\text { A alíquota principal do ICMS de cada } \\
\text { estado }\end{array}$ & Controle & CONFAZ (2018b) \\
\hline
\end{tabular}


Os modelos regressivos utilizados foram:

$$
\begin{gathered}
\text { MT }=c_{o}+\beta_{1} \text { VARDL }+\sum_{k=2}^{5} \beta_{k} \text { variáveis de controle }_{k i t}+e_{i t} \\
\text { Modelo II } \\
\text { CustoConf }=c_{o}+\beta_{1} \text { VARDL }+\sum_{k=2}^{5} \beta_{k} \text { variáveis de controle }_{k i t}+e_{i t} \\
\text { Modelo III } \\
A T=c_{o}+\beta_{1} \text { CustoConf }+\sum_{k=2}^{5} \beta_{k} \text { variáveis de controle } \text { cit }+e_{i t}
\end{gathered}
$$

\section{Apresentação, análise e discussão dos resultados}

A seguir, na Tabela 1 apresenta-se o custo de conformidade por dispositivo legal médio dos seis anos analisados. Observa-se que o Distrito Federal é o que apresenta maior custo de conformidade por dispositivo legal nos seis anos analisados, ou seja, é a Unidade Federativa onde o custo de conformidade é mais dispendioso para as empresas. Por outro lado, no estado do Piauí esse custo é menor.

Tabela 1

Custo de conformidade por dispositivo legal

\begin{tabular}{cccc}
\hline DF & 0,013006 & BA & 0,005526 \\
RJ & 0,012069 & SE & 0,005517 \\
AP & 0,011787 & MS & 0,005446 \\
ES & 0,011493 & RR & 0,005093 \\
AM & 0,010365 & MG & 0,005074 \\
SP & 0,008292 & AL & 0,004869 \\
SC & 0,007794 & TO & 0,004726 \\
RO & 0,007675 & RN & 0,004613 \\
PE & 0,007001 & MT & 0,004592 \\
GO & 0,006217 & PA & 0,004203 \\
AC & 0,005976 & PB & 0,004034 \\
RS & 0,005843 & MA & 0,003839 \\
PR & 0,005720 & PI & 0,003838 \\
CE & 0,005642 & & \\
\hline
\end{tabular}

Já na Tabela 2 é apresentada a arrecadação dos Estados, considerando a quantidade de dispositivos legais do ICMS, também ponderado pela quantidade de empresas. 
O Amazonas figura no primeiro lugar, o que quer dizer que é o Estado que arrecada mais por dispositivo legal, ponderado por empresas. Pode-se dizer que é o Estado que consegue ser mais eficiente, pois arrecada mais com menos regras tributárias. Cabe ressaltar que o Estado do Amazonas possui particularidades quanto ao ICMS em função do incentivo do imposto na Zona Franca de Manaus, apesar da coleta de dados não ter conseguido identificar essas empresas, mas fica evidente nos resultados que essa característica faz uma diferença.

Tabela 2

Arrecadação por dispositivo legal

\begin{tabular}{cccc}
\hline AM & 1,1913 & BA & 0,567 \\
PA & 0,858 & GO & 0,548 \\
DF & 0,795 & MA & 0,531 \\
RR & 0,717 & AL & 0,528 \\
PE & 0,674 & PI & 0,518 \\
ES & 0,668 & TO & 0,514 \\
RO & 0,656 & MG & 0,471 \\
SE & 0,635 & CE & 0,451 \\
PB & 0,619 & MT & 0,449 \\
AP & 0,590 & RS & 0,396 \\
AC & 0,581 & SP & 0,387 \\
MS & 0,578 & PR & 0,369 \\
RJ & 0,575 & SC & 0,354 \\
RN & 0,572 & & \\
\hline
\end{tabular}

A Tabela 3 é composta pela divisão dos resultados das Tabela 2 com a Tabela 1, pois observa a representação do custo de conformidade com arrecadação, ambos ponderados pelos dispositivos legais, ou seja, quanto custa a conformidade do ICMS para empresas em relação ao que é pago de ICMS. Esse dado evidencia o custo de conformidade refletida no produto final da transação, ou seja, o tributo recolhido. Em outras palavras, seria quanto representa o custo das obrigações acessórias em relação à obrigação principal.

Nota-se que as empresas do Estado do Rio de Janeiro gastam 0,022 com a conformidade do ICMS em relação ao imposto recolhido, ou seja, 2,2\% do imposto. E esse gasto com a conformidade varia até $0,5 \%$ nos demais Estados.

Isso evidencia que o custo de conformidade acaba sendo um incremento nos custos das organizações. Cabe ressaltar que a pesquisa não capturou os demais gastos com conformidade, como investimentos em tecnologia, ativos, consultorias e auditorias, apenas o custo com pessoas foi considerado. Esse resultado vai ao encontro da complexidade e dinamismo da legislação tributária evidenciado por Oliveira (2012), forçando as empresas a gastarem com conformidade tributária, conforme apontou estudo de Miyoshi e Nakao (2012) e afirmações feitas por Oliver e Bartley (2005). 
Tabela 3

Representação do custo de conformidade por dispositivo legal com a arrecadação por dispositivo legal

\begin{tabular}{cccc}
\hline RJ & 0,022577851 & BA & 0,009296929 \\
SP & 0,020711031 & AC & 0,009009004 \\
AP & 0,018261252 & AL & 0,008956855 \\
SC & 0,017905434 & SE & 0,008854939 \\
ES & 0,017651560 & TO & 0,008302416 \\
DF & 0,017217397 & RN & 0,008029913 \\
PR & 0,015105976 & MS & 0,007949042 \\
RS & 0,013211615 & RR & 0,006970500 \\
CE & 0,011876502 & PI & 0,006716675 \\
GO & 0,010849287 & MA & 0,006363993 \\
MG & 0,010624669 & PB & 0,006231135 \\
PE & 0,010334305 & AM & 0,005827999 \\
RO & 0,010054148 & PA & 0,005793875 \\
MT & 0,009908370 & & \\
\hline & & & \\
\hline
\end{tabular}

Observa-se assim que os Estados com maior custo de conformidade por dispositivo legal foram: Distrito Federal, Rio de Janeiro, Amapá, Espírito Santo e Amazonas; e os com menores custos foram: Piauí, Maranhão, Paraíba, Pará e Mato Grosso (Tabela 1). Os Estados com maior arrecadação por dispositivo legal foram: Amazonas, Pará, Distrito Federal, Roraima e Pernambuco, são Estados que arrecadam mais com menos regras tributárias; e os com menor arrecadação por dispositivo legal foram: Santa Catarina, Paraná, São Paulo e Rio de Janeiro (Tabela 2). Por fim, os Estados que possuem maior custo de conformidade em relação à arrecadação são: Rio de Janeiro, São Paulo, Amapá e Santa Catarina, e os Estados que possuem menor custo de conformidade em relação à obrigação principal são: Maranhão, Paraíba, Amazonas e Pará (Tabela 3); justamente os Estados com menor custo de conformidade apontados na Tabela 1.

A Tabela 4 apresenta a estatística descritiva das variáveis utilizadas neste estudo.

Tabela 4

Estatística descritiva

\begin{tabular}{lccccc}
\hline Variável & Observações & Média & Desvio-Padrão & Mínimo & Máximo \\
\hline AT & 162 & $1.37 \mathrm{e}+07$ & $2.28 \mathrm{e}+07$ & $4.21 \mathrm{e}+08$ & $1.26 \mathrm{e}+11$ \\
CustoConf & 162 & $2.10 \mathrm{e}+08$ & $4.80 \mathrm{e}+08$ & 2570647 & $2.93 \mathrm{e}+09$ \\
DispLegais & 162 & 439282.2 & 59764.6 & 333040 & 633705 \\
PIB & 162 & .0371852 & .0623199 & .002 & .326 \\
EMP & 162 & 62951.6 & 110922.2 & 1119 & 636055 \\
Alíquota & 162 & .1783333 & .0059501 & .17 & .2 \\
VARDL & 162 & .0454724 & .1114296 & -.2639087 & .2703246 \\
\hline
\end{tabular}

Em que:

AT - Arrecadação do ICMS; DispLegais - Número de Dispositivos Legais; PIB - Produto Interno Bruto; EMP - número total de empresas; Alíquota - Alíquota do ICMS; VARDL Variação dos Dispositivos Legais 
A Tabela 5 apresenta a correlação entre as variáveis.

Tabela 5

Correlação de Pearson

\begin{tabular}{lcccccc}
\hline \multicolumn{1}{c}{ AT } & CustoConf & PIB & EMP & Alíquota & VARDL \\
\hline AT & 1 & & & & & \\
CustoConf & 0.9732 & 1 & & & & \\
PIB & 0.9869 & 0.9713 & 1 & & & \\
EMP & 0.9741 & 0.9343 & 0.9709 & 1 & & \\
Alíquota & 0.2068 & 0.2273 & 0.279 & 0.1518 & 1 & \\
VARDL & -0.0706 & -0.0644 & -0.0484 & -0.0192 & 0.0025 & 1 \\
Obs = 162 & & & & & & \\
\hline
\end{tabular}

Em que:

AT - Arrecadação do ICMS; CustoConf - Custo de Conformidade; PIB - Produto Interno Bruto; EMP - número total de empresas; Alíquota - Alíquota do ICMS.

Observando a Tabela 5, nota-se uma alta correlação do PIB e o número de empresas com a arrecadação. Essa correlação é nítida, pois, por dedução, a arrecadação é impactada conforme o número de empresas e PIB. Já a alta correlação do custo de conformidade com a arrecadação, sendo ela positiva, evidencia que o aumento do custo de conformidade pelo estado gera aumento das arrecadações em $97 \%$ dos casos. Por outro lado, observa-se uma correlação negativa entre a variação dos dispositivos legais e a arrecadação, sendo um indício de que a variação dos dispositivos legais não afeta a arrecadação.

De forma geral, nota-se que as variáveis são correlacionadas quase em sua totalidade, exceto as alíquotas. Em termos estatísticos, isso indica um possível efeito de multicolinearidade dos dados, o que sugere a estimativa do modelo por métodos mais robustos ao tradicional Mínimos Quadrados Ordinários (Beck e Katz, 1995).

Para verificar se a complexidade da legislação afeta diretamente a arrecadação, realizamos a regressão linear com a equação do modelo I, estimada pelo método de Mínimos Quadrados Generalizados, com efeito fixo, para dados em painel, corrigindo possíveis problemas de autocorrelação serial e heteroscedasticidade, com resultados apresentados na Tabela 6.

Verifica-se na Tabela 6 que a proxy para complexidade da legislação, VARDL, não é significativa para explicar a arrecadação (AT).

Esse resultado sugere que a elaboração de novos dispositivos legais, aumentando a complexidade da legislação, não afeta por si só a arrecadação do governo. Esse resultado reforça a ideia de que é necessário que haja um mecanismo intermediário para fazer com que haja um aumento na arrecadação com a maior complexidade da legislação. Conforme nossas hipóteses, a suspeita é de que o custo de conformidade representa esse mecanismo. 
Tabela 6

Resultado Regressão Modelo I

\begin{tabular}{|c|c|c|c|c|c|c|c|}
\hline $\mathrm{AT}$ & Coef. & & Erro Padr. & $\mathrm{t}$ & $P>|t|$ & \multicolumn{2}{|c|}{ [Intervalo Conf. 95\%] } \\
\hline VARDL & $-6.36 \mathrm{E}+06$ & & $7.31 \mathrm{E}+06$ & -0.8700 & 0.3860 & $-2.08 \mathrm{E}+07$ & $8.08 \mathrm{E}+06$ \\
\hline PIB & $2.78 \mathrm{E}+08$ & $* * *$ & $1.91 \mathrm{E}+07$ & 14.6100 & 0.0000 & $2.41 \mathrm{E}+08$ & $3.16 \mathrm{E}+08$ \\
\hline EMP & 49750.81 & $* * *$ & 10433.83 & 4.7700 & 0.0000 & 29136.74 & 70364.87 \\
\hline Alíquota & $-1.62 \mathrm{E}+08$ & $* * *$ & $4.71 \mathrm{E}+07$ & -3.4500 & 0.0010 & $-2.55 e+11$ & $-6.93 e+10$ \\
\hline Constante & $2.94 \mathrm{E}+07$ & $* * *$ & $8.35 \mathrm{E}+06$ & 3.5200 & 0.0010 & $1.29 \mathrm{E}+07$ & $4.60 \mathrm{E}+07$ \\
\hline \multicolumn{8}{|c|}{ R-Quadrado: } \\
\hline within & 0.9847 & & & & & $\mathrm{~F}(4,152)=$ & 2448.96 \\
\hline between & 0.0000 & & & & & Prob $>\mathrm{F}=$ & 0.0000 \\
\hline overall & 0.9809 & & & & & & \\
\hline Obs.: & 162 & & & & & & \\
\hline
\end{tabular}

Em que:

AT - Arrecadação do ICMS; VARDL - Variação dos Dispositivos Legais; PIB - Produto Interno Bruto; EMP - número total de empresas; Alíquota - Alíquota do ICMS.

***, **, *: significância estatística de $1 \%, 5 \%$, e $10 \%$, respectivamente.

Dessa forma, as Tabelas 7 e 8 apresentam as regressões lineares que procuram verificar se a complexidade afeta o custo de conformidade e se este, por sua vez, afeta a arrecadação, também estimadas pelo método de Mínimos Quadrados Generalizados, com efeito fixo, para dados em painel, corrigindo possíveis problemas de autocorrelação serial e heteroscedasticidade.

A Tabelas 7 e 8 abaixo apresentam os resultados dos modelos regressivos II e III utilizados:

Tabela 7

Resultado Regressão Modelo II

\begin{tabular}{|c|c|c|c|c|c|c|c|}
\hline CustoConf & Coef. & & Erro Padr. & $\mathrm{t}$ & $\mathrm{P}>|\mathrm{t}|$ & \multicolumn{2}{|c|}{ [Intervalo Conf. 95\%] } \\
\hline VARDL & $5.44 \mathrm{E}+08$ & $* *$ & $2.49 \mathrm{E}+08$ & 2.1800 & 0.0310 & $5.18 \mathrm{E}+07$ & $1.04 \mathrm{E}+09$ \\
\hline AT & .0171671 & $* * *$ & .0026545 & 6.4700 & 0.0000 & .0119227 & .0224116 \\
\hline EMP & -1596.155 & $* * *$ & 351.7967 & -4.5400 & 0.0000 & -2291.198 & -901.112 \\
\hline PIB & $4.10 \mathrm{E}+09$ & $* * *$ & $8.33 \mathrm{E}+08$ & 4.9200 & 0.0000 & $2.45 \mathrm{E}+09$ & $5.74 \mathrm{E}+09$ \\
\hline Constante & $-1.01 \mathrm{E}+08$ & $* * *$ & $1.52 \mathrm{E}+07$ & -6.6800 & 0.0000 & $-1.31 \mathrm{E}+08$ & $-7.12 \mathrm{E}+07$ \\
\hline \multicolumn{8}{|c|}{ R-Quadrado: } \\
\hline within & 0.9602 & & & & & $\mathrm{~F}(4,152)=$ & 917.52 \\
\hline between & 0.0088 & & & & & Prob $>\mathrm{F}=$ & 0.0000 \\
\hline overall & 0.9452 & & & & & & \\
\hline Obs.: & 162 & & & & & & \\
\hline
\end{tabular}

Em que:

CustoConf - Custo de Conformidade; VARDL - Variação dos Dispositivos Legais; AT - Arrecadação do ICMS; EMP - número total de empresas; PIB - Produto Interno Bruto

***, **, *: significância estatística de $1 \%, 5 \%$, e $10 \%$, respectivamente. 
$\mathrm{Na}$ Tabela 7, nota-se que a variação dos dispositivos legais (VARDL) afeta o custo de conformidade (CustoConf), com coeficiente significativo e positivo. Esse resultado sugere que a complexidade da legislação aumenta o custo de conformidade dos contribuintes de maneira significativa, confirmando a hipótese H1. Esse resultado corrobora os resultados de Miyoshi e Nakao (2012), que apontam que as empresas incorrem em custos de conformidade para evitar sanções, e pela complexidade e dinamismo da legislação tributária destacado por Oliveira (2012).

Ainda no modelo II, observa-se o coeficiente negativo da variável empresas (EMP), indicando que quanto maior a quantidade de empresas em um estado, menor é o custo de conformidade; uma possível explicação para esse resultado é que escritórios contábeis possuem ganho de escala ao atenderem diversas empresas menores.

Tabela 8

Resultado Regressão Modelo III

\begin{tabular}{|c|c|c|c|c|c|c|c|}
\hline AT & Coef. & & Erro Padr. & $\mathrm{t}$ & $\mathrm{P}>|\mathrm{t}|$ & \multicolumn{2}{|c|}{ [Intervalo Conf. 95\%] } \\
\hline CustoConf & 10,94502 & $* * *$ & 2.022539 & 5.41 & 0.0000 & 6.949103 & 14.94094 \\
\hline PIB & $1.70 \mathrm{E}+11$ & $* * *$ & $2.65 \mathrm{E}+10$ & 6.41 & 0.0000 & $1.18 \mathrm{E}+11$ & $2.22 \mathrm{E}+11$ \\
\hline EMP & 642076 & $* * *$ & 9895.599 & 6.49 & 0.0000 & 44656.92 & 83758.27 \\
\hline Alíquota & $-8.79 \mathrm{E}+07$ & $*$ & $4.54 \mathrm{E}+07$ & -1.93 & 0.0550 & $-1.78 \mathrm{E}+11$ & $1.85 \mathrm{E}+09$ \\
\hline _cons & $1.67 \mathrm{E}+10$ & $* *$ & $8.01 \mathrm{E}+06$ & 2.08 & 0.0390 & $8.60 \mathrm{E}+08$ & $3.25 \mathrm{E}+10$ \\
\hline \multicolumn{8}{|c|}{ R-Quadrado: } \\
\hline within & 0.9871 & & & & & $\mathrm{~F}(4,152)=$ & 2913.38 \\
\hline between & 0.0061 & & & & & Prob $>F=$ & 0.0000 \\
\hline overall & 0.9838 & & & & & & \\
\hline Obs.: & 162 & & & & & & \\
\hline
\end{tabular}

Em que:

AT - Arrecadação do ICMS; CustoConf - Custo de Conformidade; PIB - Produto Interno Bruto; EMP - número total de empresas; Alíquota - Alíquota do ICMS

$* * *, * *, *$ : significância estatística de $1 \%, 5 \%$, e $10 \%$, respectivamente.

Pelo modelo III com resultados apresentados na Tabela 8, pode-se perceber que o custo de conformidade (CustoConf) afeta significativa e positivamente a arrecadação dos Estados (AT). Esse resultado sugere que os gastos das empresas com estrutura de pessoal para atender os dispositivos legais estão refletindo em maior recolhimento de tributos, confirmando a hipótese H2.

Concatenando os resultados das Tabelas 6, 7 e 8, verifica-se que a maior complexidade da legislação tributária não afeta diretamente a arrecadação, mas é capaz de afetar significativamente o custo de conformidade, sugerindo que as empresas procuram evitar sanções ao incorrerem em custos para atender ao crescimento das exigências tributárias. Isso sugere que o aumento da complexidade da legislação promove maior controle (e custo) por parte das empresas, evitando planejamento tributário e aumentando a eficiência arrecadatória, afetando assim positivamente a arrecadação. 


\section{Conclusão}

Este artigo objetivou apresentar qual é o custo de conformidade dos Estados brasileiros e examinar se a complexidade tributária afeta os custos de conformidade e se, por sua vez, estes afetam a arrecadação, aproveitando particularidades da tributação no Brasil. Para isso, nos beneficiamos da disponibilidade de dados de dispositivos legais de ICMS estabelecidos pelos Estados brasileiros e realizamos levantamentos e testes estatísticos com esses dados.

Observou-se que as empresas de todos os Estados brasileiros possuem um custo de conformidade representativo, considerando apenas a mão de obra necessária para o cumprimento das normas legais.

Os resultados dos testes sugerem que a complexidade tributária não afeta diretamente a arrecadação tributária, mas afeta positivamente os custos de conformidade das empresas, que por sua vez, afetam positivamente a arrecadação.

Esses resultados nos levam à conclusão de que é necessário que o aumento da complexidade da legislação tributária venha acompanhado de dispositivos que levem as empresas a incorrerem em custos para atender a esse aumento, para que resulte em aumento na arrecadação de tributos.

A implicação direta dessa observação é que os contribuintes reduzem sua eficiência econômica em função da necessidade de o governo proteger ou aumentar a sua arrecadação. Isso não ocorreria caso se observasse que a complexidade tributária afeta diretamente a arrecadação.

Este trabalho contribui com a literatura da área tributária ao observar a ligação entre complexidade tributária, custo de conformidade e arrecadação tributária. Ao mesmo tempo, contribui com o debate sobre a simplificação tributária e seus efeitos econômicos.

Considerando que o custo de conformidade atua como parte dos encargos tributários, que por sua vez podem afetar os indicadores econômicos de um país, este trabalho pode contribuir do ponto de vista prático ao indicar a existência dessa ligação, como argumento para a simplificação tributária e o aumento da eficiência das empresas e da economia.

A extensão dos resultados do estudo é limitada pelo recorte metodológico, considerando apenas o ICMS para o custo de conformidade tributário, que considerou apenas o período de análise entre os anos de 2011 e 2016 e as informações disponíveis nas bases de dados dos Estados. Além disso, foi considerado o percentual fixo de $54,07 \%$ das horas dos contadores, auxiliares, etc. para apuração do ICMS, o que pode variar de estado para estado, ou de profissional para profissional. Outra limitação encontra-se na impossibilidade de consideração de fatores macroeconômicos relevantes como: perfil econômico, condições de mercado e políticas de incentivos. Apenas a quantidade de empresas e PIB foram considerados para ponderações dos estados.

Sendo assim, sugere-se, para as próximas pesquisas, avaliar essa relação de custo de conformidade e arrecadação em um período maior de tempo, além de buscar informações complementares sobre os profissionais, empresas, setores de atividade e regiões dos Estados. 


\section{Referências bibliográficas}

ALLINGHAM, M. G.; SANDMO, A. Income tax evasion: a theoretical analysis. Journal of Public Economics, v. 1, n. 3-4, p. 323-338, 1972. Disponível em: https://doi.org/10.1016/00472727(72)90010-2.

BECK, N.; KATZ, J. What to do (and not to do) with time-series cross-section data. The American Political Science Review, v. 89, n. 3, p. 634-647, 1995. https://doi.org/10.2307/2082979.

BERTOLUCCI, A. V. Uma contribuição ao estudo da incidência dos custos de conformidade às leis e disposições tributárias: um panorama mundial e pesquisa dos custos das companhias de capital aberto no Brasil. Dissertação (Mestrado)-Universidade de São Paulo, São Paulo, SP, 2001.

CAVAlCANTI FILHO, J. V. O. Os custos gerados pelo Sistema Tributário Brasileiro e seus impactos no resultado econômico de um empreendimento de construção civil na cidade de Manaus. Dissertação (Mestrado)-Universidade Federal do Amazonas, Manaus, AM, 2009.

CONFAZ. Conselho Nacional de Política Fazendária. Boletim de Arrecadação. 2018. Disponível em: https://www.confaz.fazenda.gov.br/legislacao/boletim-do-icms. Acesso em:1 abr. 2018.

CONFAZ. Conselho Nacional de Política Fazendária. Alíquota ICMS Estaduais. 2018b. Disponível em: https://www.confaz.fazenda.gov.br/legislacao/aliquotas-icms-estaduais. Acesso em: 15 jan. 2018.

COX, S. P.; EGER III, R. J. Procedural complexity of tax administration: the road fund case. Journal of Public Budgeting, Accounting \& Financial Management, v. 18, n. 3, p. 259-283, 2006. Disponível em: https://doi.org/10.1108/JPBAFM-18-03-2006-B001.

DAS-GUPTA, A. Income tax compliance cost of corporations in India, 2000-01. Vikalpa, Ahmedabad, v. 31, n. 4, 2006. Disponível em: https://doi.org/10.1177/0256090920060402.

EVANS, C.; TRAN-NAM, B. Evaluating the compliance costs and benefits of the Tax Value Method: a report prepared for the Board of Taxation. Canberra, 2002. Disponível em: http://taxboard.gov.au/files/2015/07/tvm atax.pdf. Acesso em: 10 out. 2017.

FERREIRA, F. Concepção de um método de método de mensuração dos custos de conformidade tributária: o caso da EMATER/RS-ASCAR. Dissertação (Mestrado)-Universidade do Vale do Sinos, São Leopoldo, RS, 2012.

GUPTA, S.; MILLS, L. F. Does disconformity in state corporate income tax systems affect compliance cost burdens? National Tax Journal, p. 355-371, 2003. Disponível em: https://ssrn.com/abstract=386580.

IBGE. Estatística do Cadastro Central de Empresas. 2017. Disponível em: https://www.ibge.gov.br/estatisticas-novoportal/economicas/outras-estatisticas-economicas/9016estatisticas-do-cadastro-central-de-empresas.html?=\&t=o-que-e. Acesso em: 22 dez. 2017. 
IBGE. Sistema de Contas Regionais - SCR. 2017b. Disponível em: https://www.ibge.gov.br/estatisticas-novoportal/economicas/contas-nacionais/9054-contasregionais-do-brasil.html?=\&t=resultados. Acesso em: 22 dez. 2017.

KAPLOW, L. How tax complexity and enforcement affect the equity and efficiency of the income tax. National Tax Journal, Washington, v. 49, n. 1, p. 135-150, 1996. https://doi.org/10.3386/w5391.

KOPEZUK, W. Tax simplification and tax compliance: an economic perspective. In: SAWICKY, M. B. (Ed.). Bridging the tax gap. Washington D. C.: Economic Public Institute Book, 2006.

LIMA, E. S. de. Pesquisa sobre redução nos custos de conformidade tributária e os investimentos no Sistema Público de Escrituração Digital - SPED no Brasil. Dissertação (Mestrado)-Pontifícia Universidade Católica de São Paulo, São Paulo, SP, 2013.

MIYOSHI, K.; NAKAO, S. H. Riscos de conformidade tributária: um estudo de caso no Estado de São Paulo. Revista de Contabilidade e Organizações, v. 6, n. 14, p. 46-76, 2012. https://doi.org/10.11606/rco.v6i14.45400

OLIVEIRA, F. ICMS sofre 20 mudanças ao dia e atrapalha negócios. Folha de São Paulo, 27 ago. 2012.

OLIVER, T.; BARTLEY, S. Tax system complexity and compliance costs-some theoretical considerations. Economic Round-Up, 53, Winter 2005.

PAES, N. L. Mitos e verdades da política tributária brasileira. Brasília: ESAF, 2007.

PWC. Paying taxes. 2017. Disponível em: https://www.pwc.com/gx/en/paying-taxes/pdf/pwcpaying-taxes-2017-post-filing-key-findings.pdf. Acesso em: 14 maio 2018.

RECEITA FEDERAL DO BRASIL. Carga tributária no Brasil 2016. 2017. Disponível em: http://idg.receita.fazenda.gov.br/dados/receitadata/estudos-e-tributarios-e-aduaneiros/estudos-eestatisticas/carga-tributaria-no-brasil/carga-tributaria-2016.pdf. Acesso em: 10 fev. 2018.

RECEITA FEDERAL DO BRASIL. Estatísticas do Simples Nacional. 2018. Disponível em: http://www8.receita.fazenda.gov.br/SimplesNacional/Aplicacoes/ATBHE/estatisticasSinac.app/Def ault.aspx. Acesso em: 26 set. 2018.

RICHARDSON, G. Determinants of tax evasion: a cross-country investigation. Journal of International Accounting, Auditing and Taxation, v. 15, n. 2, p. 150-169, 2006.

SAAD, N. Tax knowledge, tax complexity and tax compliance: taxpayers' view. Procedia-Social and Behavioral Sciences, 109, p. 1069-1075, 2014. Disponível em: https://doi.org/10.1016/j.sbspro.2013.12.590.

SECRETARIA DO TESOURO NACIONAL. Tesouro Transparente. 2018. Disponível em: http://www.tesourotransparente.gov.br/ckan/dataset/dcaedf2. Acesso em: 15 dez. 2018. 
Otávio Gomes Cabello, Silvio Hiroshi Nakao

SLEMROD, J.; BLUMENTHAL, M. The income tax compliance cost of big business. Public Finance Quartely, v. 24, n. 4, p. 411-438, 1996. Disponível em: https://doi.org/10.1177/109114219602400401.

SLEMROD, J.; VENKATESH, V. The income tax compliance cost of large and mid-size businesses: a report to the IRS LMSB Division. Ann Arbor: University of Michigan, 2002. (Ross School of Business Working Paper, n. 914). Disponível em: https://doi.org/10.2139/ssrn.913056.

SOUZA, F. C. de, BALBINO, K. S. da S.; MIRANDA, K. F. A influência dos custos de conformidade no planejamento tributário de uma empresa comercial de produtos agrícolas. In: CONGRESSO BRASILEIRO DE CUSTOS, Uberlândia, MG, 2013. 